\title{
High Intravascular Signal Arterial Transit Time Artifacts Have Negligible Effects on Cerebral Blood Flow and Cerebrovascular Reserve Capacity Measurement Using Single Postlabel Delay Arterial Spin-Labeling in Patients with Moyamoya Disease
}

\author{
(D) M. Fahlström, (D) A. Lewén, (DP. Enblad, (D)E.-M. Larsson, and (D). Wikström
}

\begin{abstract}
BACKGROUND AND PURPOSE: Arterial spin-labeling-derived CBF values may be affected by arterial transit time artefacts. Thus, our aim was to assess to what extent arterial spin-labeling-derived CBF and cerebrovascular reserve capacity values in major vascular regions are overestimated due to the arterial transit time artifacts in patients with Moyamoya disease.
\end{abstract}

\begin{abstract}
MATERIALS AND METHODS: Eight patients with Moyamoya disease were included before or after revascularization surgery. CBF maps were acquired using a 3D pseudocontinuous arterial spin-labeling sequence, before and 5, 15, and 25 minutes after an IV acetazolamide injection and were registered to each patient's 3D-T1-weighted images. Vascular regions were defined by spatial normalization to a Montreal Neurological Institute-based vascular regional template. The arterial transit time artifacts were defined as voxels with high signal intensity corresponding to the right tail of the histogram for a given vascular region, with the cutoff selected by visual inspection. Arterial transit time artifact maps were created and applied as masks to exclude arterial transit time artifacts on CBF maps, to create corrected CBF maps. The cerebrovascular reserve capacity was calculated as CBF after acetazolamide injection relative to $\mathrm{CBF}$ at baseline for corrected and uncorrected $\mathrm{CBF}$ values, respectively.
\end{abstract}

RESULTS: A total of 16 examinations were analyzed. Arterial transit time artifacts were present mostly in the MCA, whereas the posterior cerebral artery was generally unaffected. The largest differences between corrected and uncorrected CBF and cerebrovascular reserve capacity values, reported as patient group average ratio and percentage point difference, respectively, were 0.978 (95\% Cl, 0.968-0.988) and 1.8 percentage points $(95 \% \mathrm{Cl}, 0.3-3.2$ percentage points). Both were found in the left $\mathrm{MCA}, 15$ and 5 minutes post-acetazolamide injection, respectively.

CONCLUSIONS: Arterial transit time artifacts have negligible overestimation effects on calculated vascular region-based CBF and cerebrovascular reserve capacity values derived from single-delay 3D pseudocontinuous arterial spin-labeling.

ABBREVIATIONS: $\mathrm{ACA}=$ anterior cerebral artery; $\mathrm{ACZ}=$ acetazolamide; $\mathrm{ASL}=$ arterial spin-labeling; $\mathrm{ATT}=$ arterial transit time; $\mathrm{ATT}$ over $=$ long arterial transit time where CBF is overestimated; $A T T_{\text {under }}=$ very long arterial transit time where CBF is underestimated; CVRC = cerebrovascular reserve capacity; Diff = absolute difference; MMD = Moyamoya disease; PCA = posterior cerebral artery; $\mathrm{PCASL}=$ pseudocontinuous arterial spin-labeling; PLD = postlabeling delay; $\mathrm{pp}=$ percentage points

M oyamoya disease (MMD) is characterized by stenosis or occlusion at the terminal portions of the ICA, the proximal anterior cerebral arteries (ACAs), or the MCA. Common for

Received September 29, 2019; accepted after revision December 24. From the Departments of Surgical Sciences (M.F., E.-M.L., J.W.) and Neuroscience (A.L., P.E.), Uppsala University, Uppsala, Sweden.

Please address correspondence to Markus Fahlström, MSc, Department of Surgical Sciences, Uppsala University, Akademiska Sjukhuset, SE 75185 Uppsala, Sweden; e-mail: Markus.Fahlstrom@radiol.uu.se

Indicates article with supplemental on-line tables.

Indicates article with supplemental on-line photos.

http://dx.doi.org/10.3174/ajnr.A6411 patients with MMD is the presence of abnormal vascular networks in the arterial territories close to the stenotic or occlusive lesion. ${ }^{1-3}$ Most patients can maintain adequate CBF through compensatory collateral flow, while others experience cerebral ischemia and hemorrhage. $^{1,4}$ The capability of the brain to increase CBF in response to a vasodilatory challenge can be assessed using cerebrovascular reserve capacity (CVRC) estimates, calculated as the maximal percentage increase in $\mathrm{CBF}$ after vasodilatory stimulus (commonly IV acetazolamide [ACZ], referred to as the ACZ challenge) relative to baseline. ${ }^{4}$ CVRC can predict the risk of ischemic events in patients with MMD; moreover, CVRC can also provide information on indications for or assessment after cerebral revascularization surgery. ${ }^{4,5}$ 
Arterial spin-labeling (ASL) is a completely noninvasive and highly repeatable perfusion imaging technique that has demonstrated potential in numerous cerebral disorders., ${ }^{3,6,7}$ With ASL, quantification of CBF is possible using the patient's own blood as a freely diffusible tracer. One inherent limitation with single-delay pseudocontinuous ASL (pCASL) is the dependency on an arterial transit time (ATT; ie, the time for labeled blood to flow from the labeling plane to the tissue of interest), in which arterial transit time should not exceed the user-defined parameter, postlabeling delay (PLD). This limitation is known as the ATT artifact and have 2 different effects on the derived CBF maps, depending on to what extent the blood flow is delayed (ie, the length of the ATT). First, at a very long ATT, in which no blood has reached the imaging volume in time for the readout, derived CBF will be inherently low

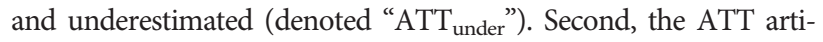
facts will appear as bright intravascular signal when the blood has reached the imaging volume but not the capillary bed-that is, it is found in precapillary arterioles, presenting as hyperintense areas in the derived $\mathrm{CBF}$ maps, thus overestimating $\mathrm{CBF}$ (denoted "ATT ${ }_{\text {over }}$ "). ${ }^{2,7-11}$ However, the $\mathrm{ATT}_{\text {over }}$ artifacts have been shown to provide important information about the presence and extent of collateral flow by visual grading in patients with MMD when long ATTs are present through collateral pathways. ${ }^{2,3,9}$ No effort has been made to quantitatively define $\mathrm{ATT}_{\text {over }}$ artifacts, and their impact on $\mathrm{CBF}$ and CVRC calculations in major vascular regions, thus, testing the hypothesis that $\mathrm{ATT}_{\text {over }}$ has negligible effects on large ROIs. ${ }^{8}$

The aim of this study was to assess to what extent vascular region-based $\mathrm{CBF}$ and CVRC are affected by the $\mathrm{ATT}_{\text {over }}$ artifacts defined by means of histogram analysis.

\section{MATERIALS AND METHODS \\ Patients}

Eight patients with confirmed MMD were included before or after revascularization surgery. This study was performed in accordance with the Declaration of Helsinki and was approved by the local ethics committee. Patients were examined by MR imaging for pre- or postoperative assessment: monitoring the progression of the disease as a decision basis for an operation or postoperative follow-up assessing the surgical outcome, compared with preoperative assessment if available, both performed on a yearly basis or close to the scheduled operation. All patients received an intravenous injection with $1 \mathrm{~g}$ or $10 \mathrm{mg} / \mathrm{kg}$ of $\mathrm{ACZ}$ for adults and children, respectively.

\section{MR Imaging Acquisition}

All examinations were performed on an Achieva 3T scanner (Philips Healthcare, Best, the Netherlands) using a 32-channel head coil. High-resolution 3D T1-weighted and 3D T2-weighted FLAIR images were acquired as structural images. A commercially available 3D-pCASL sequence with a gradient and spin-echo readout module was used for acquiring CBF maps with a PLD of $2500 \mathrm{~ms}$. The full set of acquisition parameters for all MR imaging sequences is presented in On-line Table 1. ASL-derived CBF maps were automatically calculated by the scanner according to the model defined by Buxton et $\mathrm{al}^{12}$ and recommended by Alsop et al. ${ }^{8}$ The standard ACZ challenge protocol included a total of
4 3D-pCASL acquisitions, performed before and 5, 15, and 25 minutes after the ACZ injection. Two examinations deviated from the above-described study protocol. For the first examination (the pilot), a work-in-progress 3D-pCASL sequence with a PLD of $2000 \mathrm{~ms}$ was performed before and 5, 10, and 15 minutes after ACZ injection. For the other examination, a work-in-progress 3D-pCASL sequence with a PLD of $2500 \mathrm{~ms}$ was performed with the standard ACZ challenge protocol described above. Because only 2 examinations at 10 minutes post-ACZ injections were performed, they were excluded from the statistical analysis.

\section{Vascular Regions and Image Postprocessing}

T2-weighted FLAIR images and CBF maps were coregistered to each subject's corresponding T1-weighted images. Gray matter probability maps were segmented from T1-weighted images and coregistered T2-weighted FLAIR images. ${ }^{13}$ Gray matter maps were defined with a partial volume fraction above $75 \%$. A deformation field, defining the transformation from Montreal Neurological Institute template space to subject-specific space for each subject, was derived on the basis of the subject's T1-weighted image and was applied to a standard vascular territory template, ${ }^{14,15}$ including the bilateral ACA, MCA, and posterior cerebral artery (PCA). Each subject-specific vascular territory was masked with the corresponding gray matter map to correct for partial volume effects. ${ }^{16-18}$ All processing steps, as described above, were performed using the SPM12 toolbox (http://www.fil.ion.ucl.ac.uk/spm/software/spm12).

\section{Arterial Transit Time Artifact Correction}

Arterial transit time artifacts ( $\mathrm{ATT}_{\text {over }}$ artifacts) were defined as hyperintense spots and serpiginous regions in the right tail of the corresponding histogram of all vascular territory regions and for all CBF maps, respectively (Figs 1 and 2). The right tail segment corresponding to the $\mathrm{ATT}_{\text {over }}$ artifacts was selected, including histogram bins from right to left, thus always including the highest $\mathrm{CBF}$ values. The cutoff value was selected by visual inspection so that only voxels within the hyperintense spots were included, thus creating binary $\mathrm{ATT}_{\text {over }}$ maps. Furthermore, for each vascular region, $\mathrm{ATT}_{\text {over }}$ artifact volume and $\mathrm{ATT}_{\text {over }}$ artifact ratio (defined as $\mathrm{ATT}_{\text {over }}$ volume divided by total vascular region volume) were calculated.

\section{CBF and CVRC Calculation}

All uncorrected CBF maps were smoothed using a 5-mm Gaussian filter. Corrected CBF maps were created by masking uncorrected, smoothed CBF maps with corresponding $\mathrm{ATT}_{\text {over }}$ maps, thus excluding voxels defined as $\mathrm{ATT}_{\text {over }}$ artifacts from the statistical analysis. Corrected and uncorrected $\mathrm{CBF}$ values were extracted using previously defined vascular regions, respectively. CVRC values were calculated on the basis of regional $\mathrm{CBF}$, using equation 1 for all post-ACZacquired corrected and uncorrected $\mathrm{CBF}$ maps, respectively, with corresponding $\mathrm{CBF}^{0 \text { minute }}$ defining baseline. Furthermore, the absolute difference (Diff) in percentage points between the corrected and uncorrected CVRC was calculated. Super- and subscript will be used to clarify ratios and to distinguish between baseline $(0 \mathrm{~min})$ and post- $\mathrm{ACZ}$ injection (5 min and so forth), eg, $\mathrm{CBF}_{\text {Ratio }}^{5 \mathrm{~min}}$ or $\mathrm{CVRC}_{\text {Diff }}^{5 \mathrm{~min}}$ when applicable.

AJNR Am J Neuroradiol 41:430-36 Mar 2020 www.ajnr.org 
1)

$\mathrm{CVRC}=\left(\mathrm{CBF}^{\text {post }-\mathrm{ACZ}}-\mathrm{CBF}^{0 \text { minute }}\right) /\left(\mathrm{CBF}^{0 \text { minute }}\right)$

\section{Statistical Analysis}

For descriptive analysis of $\mathrm{ATT}_{\text {over }}$ volume and $\mathrm{ATT}_{\text {over }}$ ratio, mean, maximum value, and $95 \%$ CI were calculated. Further-

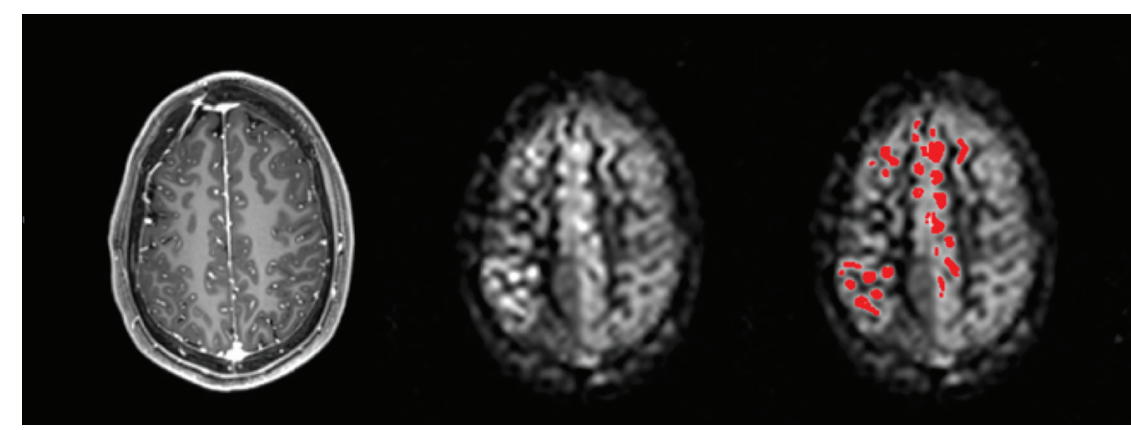

more, $\mathrm{CBF}$ and CVRC comparisons were performed by descriptive analysis of the corrected-to-uncorrected ratio $(\mathrm{CBF})$ or the absolute difference in percentage points (CVRC); thus, the average ratio/difference with $95 \% \mathrm{CI}$ and maximum/minimum were calculated and analyzed. Descriptive analysis was performed for all vascular regions and baseline and 5, 15, and 25 minutes post-ACZ injection, respectively. A correlation analysis was peformed comparing the $\mathrm{CBF}_{\text {Ratio }}$ with ATT volume using the Pearson correlation coefficient. This was performed using all time point values as 1 large dataset. Derived $P$ values are presented as exact values and are 2-sided, and $P<.05$ is considered significant. GraphPad Prism 8 for Mac (GraphPad Software, San Diego, California) was used for statistical analysis and graph design.

FIG 1. A 3D T1-weighted image with derived CBF maps with and without binary $A T_{\text {over }}$ voxels (red).

Left ACA

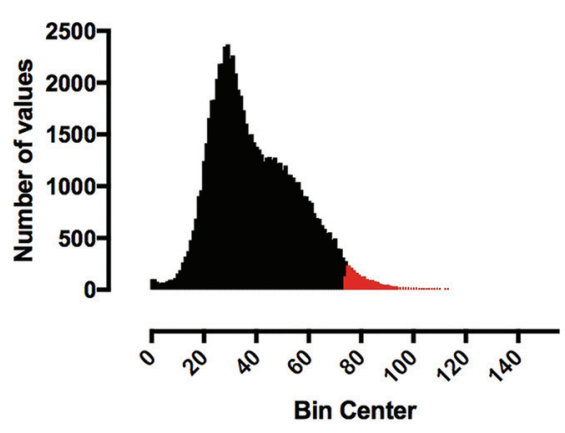

Left MCA

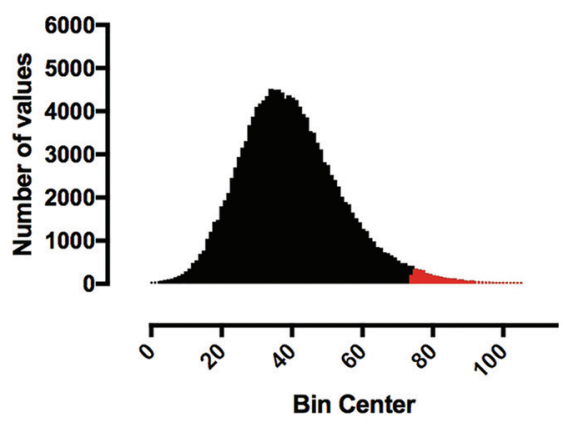

Left PCA

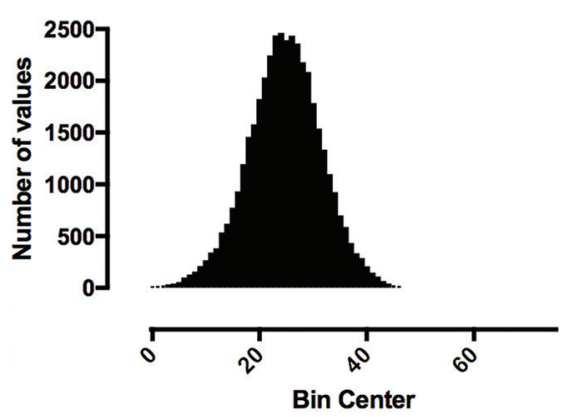

Right ACA

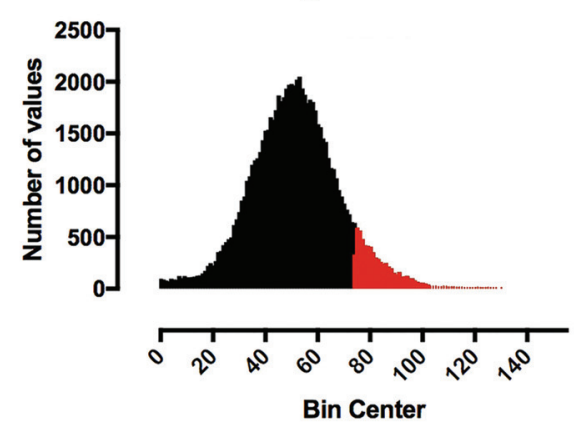

Right MCA

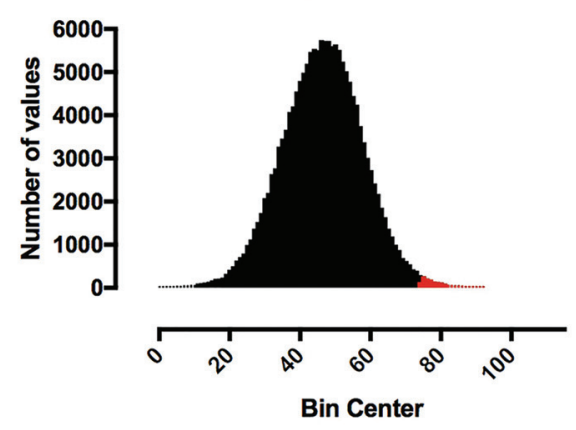

Right PCA

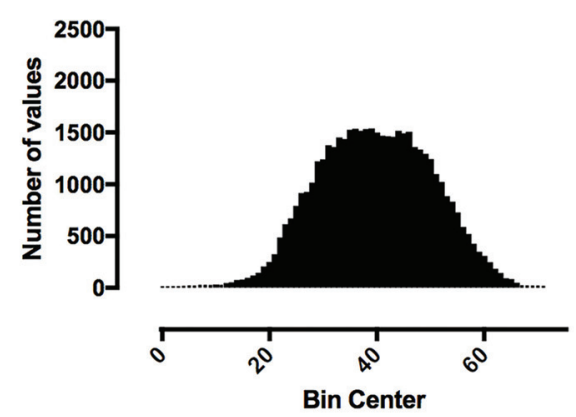

CBF

ATT Artifact

CBF

ATT Artifact

CBF
CBF

ATT Artifact

FIG 2. Derived histograms and defined $A T_{\text {over }}$ voxels from the CBF maps presented in Fig 1. 
Table 1: Average of derived ratios, minimum/maximum, and $95 \% \mathrm{Cl}$ for $\mathrm{CBF}_{\text {Ratio }}$

\begin{tabular}{|c|c|c|c|c|c|c|}
\hline \multirow[b]{2}{*}{$\mathrm{CBF}_{\text {Ratio }}$} & \multicolumn{2}{|c|}{ ACA } & \multicolumn{2}{|c|}{ MCA } & \multicolumn{2}{|c|}{ PCA } \\
\hline & Left & Right & Left & Right & Left & Right \\
\hline \multicolumn{7}{|l|}{ Baseline } \\
\hline Mean & 0.989 & 0.986 & 0.979 & 0.980 & 0.995 & 0.996 \\
\hline $95 \% \mathrm{Cl}$ & $0.981-0.995$ & $0.978-0.994$ & $0.971-0.988$ & $0.972-0.987$ & $0.990-1.000$ & $0.992-1.001$ \\
\hline Min-max & 0.949-1.000 & $0.942-1.000$ & $0.954-1.000$ & $0.958-0.999$ & $0.961-1.000$ & $0.970-1.000$ \\
\hline \multicolumn{7}{|l|}{5 minutes } \\
\hline Mean & 0.991 & 0.987 & 0.981 & 0.978 & 0.992 & 0.992 \\
\hline $95 \% \mathrm{Cl}$ & $0.986-0.991$ & $0.980-0.995$ & $0.975-0.988$ & $0.970-0.986$ & $0.985-0.999$ & $0.985-0.999$ \\
\hline Min-max & $0.975-0.999$ & $0.955-0.998$ & $0.961-0.998$ & $0.950-0.998$ & $0.956-1.000$ & $0.952-1.000$ \\
\hline \multicolumn{7}{|l|}{15 minutes } \\
\hline Mean & 0.990 & 0.987 & 0.981 & 0.979 & 0.995 & 0.996 \\
\hline $95 \% \mathrm{Cl}$ & $0.983-0.997$ & $0.980-0.993$ & $0.973-0.988$ & $0.970-0.987$ & $0.992-0.998$ & $0.993-0.999$ \\
\hline Min-max & $0.948-0.999$ & $0.957-0.999$ & $0.937-0.998$ & $0.942-0.997$ & 0.980-1.000 & $0.982-1.000$ \\
\hline \multicolumn{7}{|l|}{25 minutes } \\
\hline Mean & 0.989 & 0.985 & 0.980 & 0.978 & 0.995 & 0.997 \\
\hline $95 \% \mathrm{Cl}$ & $0.981-0.998$ & $0.977-0.993$ & $0.970-0.990$ & $0.968-0.988$ & 0.991-0.999 & $0.994-1.000$ \\
\hline Min-max & $0.943-1.000$ & $0.960-1.000$ & $0.938-0.996$ & $0.944-0.997$ & $0.976-1.000$ & $0.982-1.000$ \\
\hline
\end{tabular}

Note:-Min-max indicates minimum-maximum.

Table 2: Average of derived differences, maximum, and $95 \% \mathrm{Cl}$ for $\mathrm{CVRC}_{\mathrm{Diff}}$

\begin{tabular}{|c|c|c|c|c|c|c|}
\hline \multirow[b]{2}{*}{ CVRC $_{\text {Diff }}(p p)$} & \multicolumn{2}{|c|}{$\mathrm{ACA}$} & \multicolumn{2}{|c|}{ MCA } & \multicolumn{2}{|c|}{ PCA } \\
\hline & Left & Right & Left & Right & Left & Right \\
\hline \multicolumn{7}{|l|}{5 minutes } \\
\hline Mean & 0.7 & 1.1 & 1.8 & 1.7 & 0.8 & 1.5 \\
\hline $95 \% \mathrm{Cl}$ & $0.3-1.0$ & $0.4-1.7$ & $0.3-3.2$ & $0.9-2.6$ & $0.1-1.6$ & $-0.5-3.1$ \\
\hline Maximum & 2.2 & 4.2 & 11.3 & 5.9 & 5.2 & 7.8 \\
\hline \multicolumn{7}{|l|}{15 minutes } \\
\hline Mean & 0.8 & 1.1 & 0.9 & 1.5 & 0.9 & 0.8 \\
\hline $95 \% \mathrm{Cl}$ & $0.3-1.2$ & $0.4-1.7$ & $0.4-1.5$ & $0.6-2.3$ & $-0.2-1.9$ & $-0.2-1.9$ \\
\hline Maximum & 3.5 & 4.5 & 3.1 & 5.4 & 6.7 & 6.8 \\
\hline \multicolumn{7}{|l|}{25 minutes } \\
\hline Mean & 1.0 & 1.3 & 1.7 & 1.5 & 0.3 & 0.4 \\
\hline $95 \% \mathrm{Cl}$ & $0.4-1.7$ & $0.4-2.2$ & $0.5-2.9$ & $0.7-2.3$ & $0.0-0.5$ & $-0.2-1.1$ \\
\hline Maximum & 4.1 & 5.5 & 5.8 & 4 & 0.9 & 3.3 \\
\hline
\end{tabular}

injection)-that is, the largest difference found between any corrected and uncorrected $\mathrm{CBF}$ values was $6.3 \%$ (Table 1 and On-line Fig 2). The correlation between the $\mathrm{CBF}_{\text {Ratio }}$ and ATT volume was negative and strong $(r=-0.85, P<.001$, On-line Fig 3$)$.

\section{Comparison of CVRC Values}

For all major vascular regions, including ACZ challenges, the calculated average $\mathrm{CVRC}_{\text {Diff }}$ was small, with a narrow 95\% CI (highest CVRC Diff $_{\text {av- }}$ erage, 1.8 percentage points [pp]; $95 \%$ CI, 0.3-3.2 pp; On-line Fig 4). The

\section{RESULTS}

\section{Patients}

A total of 16 examinations were performed, which included 9 preoperative and 7 postoperative examinations. Two patients had both pre- and postoperative examinations.

\section{ATT over Volume}

A representative example of $\mathrm{ATT}_{\text {over }}$ artifact definition can be seen in Figs 1 and 2. For all examinations, arranged by post-ACZ injection time points, descriptive statistics for bilateral ACA, MCA, and PCA are presented in On-line Table 2 and On-line Fig 1. The largest $\mathrm{ATT}_{\text {over }}$ artifact volume was $30.9 \mathrm{~cm}^{3}$ (left MCA at 25 minutes post-ACZ injection). Generally, $\mathrm{ATT}_{\text {over }}$ artifacts were mostly present in the MCA, while the PCA was mostly unaffected; furthermore, the volumes typically increased from baseline compared with post-ACZ injection.

\section{Comparison of CBF Values}

The average $\mathrm{CBF}_{\text {Ratio }}$ was generally close to 1.0 , with a narrow 95\% CI, considering all major vascular regions including ACZ challenges, thus suggesting minor differences between corrected and uncorrected CBF. The smallest calculated $\mathrm{CBF}_{\text {Ratio }}$ was 0.937 (corrected CBF, $51.16 \mathrm{~mL} / 100 \mathrm{~g} / \mathrm{min}$, and uncorrected, 54.61 $\mathrm{mL} / 100 \mathrm{~g} / \mathrm{min}$ in the left MCA at 15 minutes post-ACZ maximum CVRC $_{\text {Diff }}$ was 11.3 pp (the corrected CVRC was $46.2 \%$ and the uncorrected CVRC was $34.9 \%$ in the left MCA at 5 minutes post-ACZ injection, Table 2).

\section{DISCUSSION}

This study assessed the extent of the $\mathrm{ATT}_{\text {over }}$ artifacts, presenting as hyperintense spots on single-delay ASL acquisitions during ACZ challenges in patients with MMD, and the effect of the $\mathrm{ATT}_{\text {over }}$ artifacts on CBF and CVRC values in major vascular territories. Major findings included the following: In major vascular territories, the $\mathrm{ATT}_{\text {over }}$ artifacts have negligible effects on derived CBF and CVRC values.

The anterior circulation is predominantly affected in patients with $M M D ;{ }^{1,4,9}$ furthermore, no patient in our study had any occlusion affecting the PCA. This finding agrees with the derived distribution of average $\mathrm{ATT}_{\text {over }}$ volume in the bilateral major vascular territories used in this study.

Comparing corrected and uncorrected $\mathrm{CBF}$ values, we found small, negligible differences. Alsop et $\mathrm{al}^{8}$ suggested that CBF measurements may still be valid in large ROIs in the presence of $\mathrm{ATT}_{\text {over }}$ if flow-crushing gradients are not used. The results of this study prove this hypothesis valid for major vascular regions in patients with MMD. Furthermore, because CVRC is an 
imperative prognostic factor for assessing and evaluating patients with MMD pre- and postsurgery, ${ }^{4,5}$ assessing how CVRC is affected by the $\mathrm{ATT}_{\text {over }}$ artifacts is of great importance. We found generally small, negligible percentage point differences between corrected and uncorrected CVRC. The maximum difference was 11.3 pp (corrected CVRC, 46.2\%; and uncorrected CVRC, $34.9 \%)$. In this case, the difference was found at 5 minutes postACZ injection, a time point not commonly used during ACZ challenges and CVRC calculation. ${ }^{4,19-24} \mathrm{We}$ also assessed possible correlations between $\mathrm{CBF}_{\text {Ratio }}$ and $\mathrm{ATT}_{\text {over }}$ volume and found a strong, significant negative correlation $(r=-0.85, P<.001)$. This is expected; as we exclude more pixel values with increasing ATT volume, the effect on average corrected mean CBF would inherently be larger. However, we do not consider this relationship to be a potential bias.

In a quantitative regional analysis comparing multidelay pulsed ASL with DSC MR imaging, Martin et $\mathrm{al}^{25}$ found a moderate correlation for normalized CBF, which increased slightly after exclusion of $\mathrm{ATT}_{\text {over }}$ in the MCA region. It is inherently difficult to compare these results with ours because important inconsistencies between the studies exist. The study by Martin et al was primarily designed to compare a multidelay pulsed ASL with DSC MR imaging in patients with unilateral atherosclerotic stenoocclusive disease. CBF was normalized to contralateral vascular regions not affected by occlusion, and pulsed ASL has a lower achievable SNR compared with pCASL. Multidelay ASL has been proposed as an imaging technique for patients with MMD, due to mainly 1 advantage: less vulnerability to ATT artifacts. On the other hand, single-delay ASL has signal-to-noise and scan time advantages over multidelay ASL, ${ }^{26}$ and the results presented here should be considered favorable for single-delay ASL.

We acquired $\mathrm{CBF}$ measurements 5, 15, and 25 minutes post$\mathrm{ACZ}$ injection. While the $\mathrm{ATT}_{\text {over }}$ volume increased post-ACZ injection, there were no pronounced effects on either CBF or CVRC. The increase in $\mathrm{ATT}_{\text {over }}$ volume is expected, considering the reported increase in MTT derived from perfusion CT postACZ injection, especially in regions supplied by occluded vessels. ${ }^{4,27}$ However, Federau et $\mathrm{al}^{21}$ showed that ATT estimates based on multidelay ASL-acquired data decreased after injection of ACZ.

The current reference standard for CBF measurements (and thus CVRC) is ${ }^{15} \mathrm{O}$-water PET. $9,24{ }^{15} \mathrm{O}$-water PET requires, however, an on-site cyclotron, and the availability is limited. ${ }^{9}$ SPECT is more readily available and is frequently used for MMD evaluation, but with limitations such as poor spatial resolution. ${ }^{24,28}$ In addition, both PET and SPECT imaging are invasive and expose the patient to ionizing radiation. $2,5,9,23,29$ Consequently, assessing the correlation between ASL-derived $\mathrm{CBF}$ and $\mathrm{CBF}$ derived from PET and SPECT is important, and this has been studied extensively. High correlations have been reported between SPECT $\left({ }^{123} \mathrm{I}\right.$-iodoamphetamine and ${ }^{99 \mathrm{~m}} \mathrm{Tc}$ hexamethylpropyleneamine oxime) and ASL (different sequences, PLDs, and field strengths). ${ }^{5,23,24,30}$ The dependency of the PLD as a parameter affecting correlation with ${ }^{15} \mathrm{O}$-water PET has been highlighted by others. Hara et $\mathrm{al}^{29}$ concluded that ASL acquired with a short PLD (1525 ms) showed better correlation with ${ }^{15} \mathrm{O}$-gas $\mathrm{PET}$ in normally perfused areas compared with a long PLD $(2525 \mathrm{~ms})$ in patients with MMD. However, in symptomatic areas, where arterial transit is delayed, correlation increased for a long PLD and was then higher than a short PLD. The importance of the PLD was furthermore highlighted in a study by Inoue et $\mathrm{al}^{6}{ }^{6}$ presenting different CVRC values in healthy volunteers depending on the PLD. In a comparative study using simultaneously acquired ASL and ${ }^{15} \mathrm{O}$-water PET, Fan et $\mathrm{al}^{9}$ concluded that long PLDs (4000 ms, compared with our PLD of $2500 \mathrm{~ms}$ ) are needed to achieve good correlations between relative CBF measurements in patients with MMD.

Another limitation addressed by the authors is the lack of acquired arterial blood samples to enable absolute CBF from kinetic modeling of the PET data. Arterial blood samples were not collected, as stated by the authors, to minimize patient discomfort. Goetti et $\mathrm{al}^{31}$ also reported good correlations in children and young adults with MMD between ASL and ${ }^{15} \mathrm{O}$-water PET; however, data were not acquired simultaneously, with a short PLD of $1500 \mathrm{~ms}$ and after normalization to cerebellar CBF-values. Again, no arterial blood samples were collected. This feature is an important limitation with ${ }^{15} \mathrm{O}$-water PET; quantitative studies require an arterial input function most reliably acquired through continuous arterial sampling. ${ }^{32,33}$ Arterial sampling may not be feasible in patients in poor condition and is not feasible in children with MMD. ${ }^{33}$ However, several noninvasive methods can estimate relative $\mathrm{CBF}$ in the absence of existing arterial blood sampling. ${ }^{33,34}$ ASL is, by definition, fully noninvasive, with no radiation exposure and greater availability than cyclotron-dependent ${ }^{15} \mathrm{O}$-water PET. As concluded by other authors, ASLderived $\mathrm{CBF} / \mathrm{CVRC}$ has potential in pre-/postoperative assessment in patients with MMD. ${ }^{3,5,6,9,24-26,29,31,35-37}$

\section{Limitations}

In the histogram analysis, the right tail cutoff value defining the $\mathrm{ATT}_{\text {over }}$ artifacts was selected by visual inspection. A manual approach with only 1 reader is generally considered a limitation due to the dependency on the reader's ability to achieve high specificity. However, the histogram analysis method appeared robust, and the highest $\mathrm{CBF}$ values were always included because the selection of the $\mathrm{ATT}_{\text {over }}$ segment of the histogram was performed from right to left; thus, we do not consider this to confound our results.

Another drawback of our study was that we did not have a sufficient imaging slab to cover the whole brain, including the cerebellum, which has been used for normalization in several publications. $^{2,24,29,31}$ However, cerebellar normalization is not commonly performed, and CVRC is, by definition, a ratio; thus, we do not expect this to affect our results.

Two ACZ challenges were performed with a work-in-progress 3D-pCASL sequence with PLDs of 2000 and $2500 \mathrm{~ms}$, respectively. These examinations were not excluded from this study and were not different from the others in regard to the derived $\mathrm{CBF}_{\text {Ratio }}$ and $\mathrm{CVRC}_{\text {Diff. }}$

In the present study, no effort was made to assess whether the presence of $\mathrm{ATT}_{\text {under }}$ artifacts would affect our results. Given the theory of ATT artifacts as outlined in the introduction, the presence of $\mathrm{ATT}_{\text {over }}$ artifacts could indicate that possible surrounding regional hypoperfusion can be a consequence of $\mathrm{ATT}_{\text {under }}$ artifacts. Still, we are analyzing ratio-based data, and given that both corrected and uncorrected CBF maps would have the same extent 
for the $\mathrm{ATT}_{\text {under }}$ artifacts, we consider this to have a minor impact on our results and conclusions. Moreover, the use of a long PLD in this study will reduce the $\mathrm{ATT}_{\text {under }}$ artifacts as reported by Hara et al. ${ }^{29}$

Partial volume effects are caused by the limited spatial resolution in ASL and tend to result in under- and overestimation of $\mathrm{CBF}$ in the GM and WM, respectively. ${ }^{17,38}$ We used a simple partial volume correction method, masking all vascular regions with a GM mask. Furthermore, to reduce any registration errors between pre- and post-ACZ ASL acquisitions, we applied a Gaussian filter of $5 \mathrm{~mm}$ before $\mathrm{ATT}_{\text {over }}$ correction. ${ }^{5,20,31,35}$

We argue that single-delay ASL still has potential for this patient population. We also argue that ASL, in general, should be considered an excellent method for clinical assessment in patients with MMD. ASL allows us to capture the dynamic response ${ }^{6}$ and further explore the clinical implications of the ACZ challenge in patients with MMD. Still, further studies are needed to evaluate single-delay and as well as multidelay ASL as a complete substitute for other, invasive, not readily available, ionizing radiationbased methods.

\section{CONCLUSIONS}

$\mathrm{ATT}_{\text {over }}$ artifacts, defined by means of histogram analysis, have negligible effects on calculated $\mathrm{CBF}$ and CVRC derived from single-delay 3D-pCASL while suppressing $\mathrm{ATT}_{\text {under }}$ using a long PLD. Major vascular regions are large enough for $\mathrm{CBF}$ and CVRC to be valid, thus making single-delay 3D-pCASL a valuable tool in pre- and postoperative assessment of patients with MMD.

\section{REFERENCES}

1. Kuroda S, Houkin K. Moyamoya disease: current concepts and future perspectives. Lancet Neurol 2008;7:1056-66 CrossRef Medline

2. Lee S, Yun TJ, Yoo RE, et al. Monitoring cerebral perfusion changes after revascularization in patients with Moyamoya disease by using arterial spin-labeling MR imaging. Radiology 2018;288:565-72 CrossRef Medline

3. Zaharchuk G, Do HM, Marks MP, et al. Arterial spin-labeling MRI can identify the presence and intensity of collateral perfusion in patients with Moyamoya disease. Stroke 2011;42:2485-91 CrossRef Medline

4. Vagal AS, Leach JL, Fernandez-Ulloa M, et al. The acetazolamide challenge: techniques and applications in the evaluation of chronic cerebral ischemia. AJNR Am J Neuroradiol 2009;30:876-84 CrossRef Medline

5. Noguchi T, Kawashima M, Nishihara M, et al. Noninvasive method for mapping CVR in Moyamoya disease using ASL-MRI. Eur J Radiol 2015;84:1137-43 CrossRef Medline

6. Inoue $\mathrm{Y}$, Tanaka $\mathrm{Y}$, Hata $\mathrm{H}$, et al. Arterial spin-labeling evaluation of cerebrovascular reactivity to acetazolamide in healthy subjects. AJNR Am J Neuroradiol 2014;35:1111-16 CrossRef Medline

7. Zaharchuk G. Arterial spin-labeling for acute stroke: practical considerations. Transl Stroke Res 2012;3:228-35 CrossRef Medline

8. Alsop DC, Detre JA, Golay X, et al. Recommended implementation of arterial spin-labeled perfusion MRI for clinical applications: a consensus of the ISMRM Perfusion Study Group and the European Consortium for ASL in Dementia. Magn Reson Med 2015;73:102-16 CrossRef Medline

9. Fan AP, Guo J, Khalighi MM, et al. Long-delay arterial spin labeling provides more accurate cerebral blood flow measurements in
Moyamoya patients: a simultaneous positron emission tomography/MRI study. Stroke 2017;48:2441-49 CrossRef Medline

10. Mutke MA, Madai VI, von Samson-Himmelstjerna FC, et al. Clinical evaluation of an arterial-spin-labeling product sequence in stenoocclusive disease of the brain. PLoS One 2014;9:e87143 CrossRef Medline

11. Chng SM, Petersen ET, Zimine I, et al. Territorial arterial spin labeling in the assessment of collateral circulation: comparison with digital subtraction angiography. Stroke 2008;39:3248-54 CrossRef Medline

12. Buxton RB, Frank LR, Wong EC, et al. A general kinetic model for quantitative perfusion imaging with arterial spin-labeling. Magn Reson Med 1998;40:383-96 CrossRef Medline

13. Lindig T, Kotikalapudi R, Schweikardt D, et al. Evaluation of multimodal segmentation based on 3D T1-, T2- and FLAIR-weighted images: the difficulty of choosing. Neuroimage 2018;170:210-21 CrossRef Medline

14. Mutsaerts HJ, van Dalen JW, Heijtel DF, et al. Cerebral perfusion measurements in elderly with hypertension using arterial-spin labeling. PLoS One 2015;10:e0133717 CrossRef Medline

15. Tatu L, Moulin T, Bogousslavsky J, et al. Arterial territories of the human brain: cerebral hemispheres. Neurology 1998;50:1699-1708 CrossRef Medline

16. Chen Y, Wang DJ, Detre JA. Test-retest reliability of arterial spinlabeling with common labeling strategies. J Magn Reson Imaging 2011;33:940-49 CrossRef Medline

17. Petr J, Schramm G, Hofheinz F, et al. Partial volume correction in arterial spin-labeling using a Look-Locker sequence. Magn Reson Med 2013;70:1535-43 CrossRef Medline

18. Wu WC, Jiang SF, Yang SC, et al. Pseudocontinuous arterial spin labeling perfusion magnetic resonance imaging: a normative study of reproducibility in the human brain. Neuroimage 2011;56:124450 CrossRef Medline

19. Andaluz N, Choutka O, Vagal A, et al. Patient selection for revascularization procedures in adult Moyamoya disease based on dynamic perfusion computerized tomography with acetazolamide challenge (PCTA). Neurosurg Rev 2010;33:225-32; discussion 23233 CrossRef Medline

20. Detre JA, Samuels OB, Alsop DC, et al. Noninvasive magnetic resonance imaging evaluation of cerebral blood flow with acetazolamide challenge in patients with cerebrovascular stenosis. J Magn Reson Imaging 1999;10:870-75 CrossRef Medline

21. Federau C, Christensen S, Zun Z, et al. Cerebral blood flow, transit time, and apparent diffusion coefficient in Moyamoya disease before and after acetazolamide. Neuroradiology 2017;59:5-12 CrossRef Medline

22. Ni WW, Christen T, Rosenberg J, et al. Imaging of cerebrovascular reserve and oxygenation in Moyamoya disease. J Cereb Blood Flow Metab 2017;37:1213-22 CrossRef Medline

23. Noguchi T, Kawashima M, Irie H, et al. Arterial spin-labeling MR imaging in moyamoya disease compared with SPECT imaging. Eur J Radiol 2011;80:e557-62 CrossRef Medline

24. Yun TJ, Paeng JC, Sohn CH, et al. Monitoring cerebrovascular reactivity through the use of arterial spin labeling in patients with Moyamoya disease. Radiology 2016;278:205-13 CrossRef Medline

25. Martin SZ, Madai VI, von Samson-Himmelstjerna FC, et al. 3D GRASE pulsed arterial spin labeling at multiple inflow times in patients with long arterial transit times: comparison with dynamic susceptibility-weighted contrast-enhanced MRI at 3 Tesla. J Cereb Blood Flow Metab 2015;35:392-401 CrossRef Medline

26. Donahue MJ, Achten E, Cogswell PM, et al. Consensus statement on current and emerging methods for the diagnosis and evaluation of cerebrovascular disease. J Cereb Blood Flow Metab 2018;38:13911417 CrossRef Medline

27. Eskey CJ, Sanelli PC. Perfusion imaging of cerebrovascular reserve. Neuroimaging Clin N Am 2005;15:367-81, xi CrossRef Medline 
28. Lee M, Zaharchuk G, Guzman R, et al. Quantitative hemodynamic studies in Moyamoya disease: a review. Neurosurg Focus 2009;26:E5 CrossRef Medline

29. Hara S, Tanaka Y, Ueda Y, et al. Noninvasive evaluation of CBF and perfusion delay of Moyamoya disease using arterial spin-labeling MRI with multiple postlabeling delays: comparison with (15)OGas PET and DSC-MRI. AJNR Am J Neuroradiol 2017;38:696-702 CrossRef Medline

30. Sugino T, Mikami T, Miyata K, et al. Arterial spin-labeling magnetic resonance imaging after revascularization of Moyamoya disease. $J$ Stroke Cerebrovasc Dis 2013;22:811-16 CrossRef Medline

31. Goetti R, Warnock G, Kuhn FP, et al. Quantitative cerebral perfusion imaging in children and young adults with Moyamoya disease: comparison of arterial spin-labeling-MRI and $\mathrm{H}(2)[(15) \mathrm{O}]-$ PET. AJNR Am J Neuroradiol 2014;35:1022-28 CrossRef Medline

32. Boellaard R, van Lingen A, van Balen SC, et al. Characteristics of a new fully programmable blood sampling device for monitoring blood radioactivity during PET. Eur J Nucl Med 2001;28:81-89 CrossRef Medline

33. Koopman T, Yaqub M, Heijtel DF, et al. Semi-quantitative cerebral blood flow parameters derived from non-invasive $[(15) \mathrm{O}] \mathrm{H} 2 \mathrm{O}$
PET studies. J Cereb Blood Flow Metab 2019;39:163-72 CrossRef Medline

34. Khalighi MM, Deller TW, Fan AP, et al. Image-derived input function estimation on a TOF-enabled PET/MR for cerebral blood flow mapping. J Cereb Blood Flow Metab 2018;38:126-35 CrossRef Medline

35. Blauwblomme T, Lemaitre H, Naggara O, et al. Cerebral blood flow improvement after indirect revascularization for pediatric Moyamoya disease: a statistical analysis of arterial spin-labeling MRI. AJNR Am J Neuroradiol 2016;37:706-12 CrossRef Medline

36. Wang R, Yu S, Alger JR, et al. Multi-delay arterial spin labeling perfusion MRI in Moyamoya disease-comparison with CT perfusion imaging. Eur Radiol 2014;24:1135-44 CrossRef Medline

37. Choi HJ, Sohn $\mathrm{CH}$, You $\mathrm{SH}$, et al. Can arterial spin-labeling with multiple postlabeling delays predict cerebrovascular reserve? AJNR Am J Neuroradiol 2018;39:84-90 CrossRef Medline

38. Ahlgren A, Wirestam R, Lind E, et al. A linear mixed perfusion model for tissue partial volume correction of perfusion estimates in dynamic susceptibility contrast MRI: impact on absolute quantification, repeatability, and agreement with pseudo-continuous arterial spin labeling. Magn Reson Med 2017;77:2203-14 CrossRef Medline 